\title{
INICIATIVAS DE COMUNICAÇÃO NA INTERAÇÃO ENTRE CRIANÇAS COM DISTÚRBIOS DO ESPECTRO AUTÍSTICO E SUAS MÃES: ANÁLISE PRAGMÁTICA
}

\section{Communication initiatives in the interaction among children with autistic spectrum disorders and their mothers: pragmatic analysis}

\author{
Maria Cláudia Brito ${ }^{(1)}$, Andréa Regina Nunes Misquiatti (2)
}

\begin{abstract}
RESUMO
Objetivos: investigar as iniciativas de comunicação na interação entre crianças com distúrbios do espectro autístico (DEA) e suas mães, por meio de uma análise pragmática. Método: cinco crianças com DEA, de ambos os gêneros, idades entre cinco e 12 anos juntamente com suas mães. $O$ grupo controle constou de cinco crianças com desenvolvimento normal, pareadas com as crianças com DEA de acordo com gênero e idade e suas respectivas mães, totalizando 20 participantes. Para a caracterização da amostra foram utilizadas uma Ficha informativa e a Escala de Avaliação de Traços Autísticos. Cada díade foi filmada por 30 minutos durante as interações lúdicas, analisadas a partir do Protocolo de Pragmática. Este instrumento avalia as iniciativas de comunicação, meios e funções comunicativas expressas. Os dados foram tratados estatisticamente $(p<0,05$; foram utilizados os testes não-paramétricos de Wilcoxon e de Mann-Whitney). Resultados: foram observadas diferenças estatisticamente significantes entre as iniciativas comunicativas das crianças com DEA e suas mães, quando comparadas ao grupo controle. As díades que envolveram crianças com DEA iniciaram menos a comunicação e utilizaram predominantemente gestos e vocalizações menos interativas. Conclusões: estes dados possibilitaram descrever aspectos fundamentais para compreensão das manifestações clínicas da população estudada e para a elaboração de programas de intervenção que visem melhorar a qualidade de vida da criança e sua família.
\end{abstract}

DESCRITORES: Relações Mãe-Filho; Transtorno Autístico; Comunicação; Estudos de Intervenção

\section{INTRODUÇÃO}

As medidas de intervenção fonoaudiológicas que se referem às alterações da linguagem infantil, devem fundamentalmente envolver a atuação sistemática junto às famílias, para promover efetivamente o desenvolvimento da criança e melhora nas interações entre pais e filhos. Pesquisadores descrevem diversas implicações nas interações

(1) Fonoaudióloga, Pesquisadora Colaboradora; Doutora em Educação e Pós-Doutoranda em Educação Especial pela Universidade Estadual Paulista, UNESP, Marília, SP, Brasil

(2) Fonoaudióloga; Professora do Departamento de Fonoaudiologia da Universidade Estadual Paulista - UNESP, Marília, São Paulo, Brasil; Doutora em Linguística pela Faculdade de Filosofia, Letras e Ciências Humanas da Universidade de São Paulo.

Conflito de interesses: inexistente entre mães e filhos para a saúde física e mental de ambos ${ }^{1}$. Portanto, os aspectos dessas interações relacionadas ao desenvolvimento social, afetivo, linguístico e cognitivo da criança $a^{2,3}$, devem ser considerados no estudo da organização comportamental e competências da criança ${ }^{4}$. Além disso, é necessário o enfoque na importância das relações mãe-filho para a mulher, tema mais restritamente explorado, embora possa trazer grandes implicações à vida das mesmas, principalmente quando a criança apresenta um distúrbio de desenvolvimento. Entre os distúrbios de desenvolvimento que tem sido apontados como bastante impactantes nas interações entre mães e filhos, estão os distúrbios do espectro autístico (DEA).

Os DEA se referem a um subgrupo de indivíduos que compartilham as principais características do autismo, com diferentes graus de severidade ${ }^{5}$. 
Estes transtornos são caracterizados por comprometimentos nas áreas da interação social, comunicação e presença de comportamentos e interesses restritos e estereotipados.

Embora sejam rejeitadas as premissas de que pais de crianças autistas são os agentes causadores da deficiência do filho ${ }^{6}$, o comportamento afetivo incomum dessas crianças durante a interação, parece exercer influência negativa sobre a expressão emocional materna ${ }^{7}$.

A literatura indica que os pais de crianças com DEA apresentam altas taxas de depressão e ansiedade $^{8}$ e sugere que há relação entre os níveis de tensão apresentados pela mãe e o grau de severidade da sintomatologia manifestada pela criança ${ }^{8,9}$. Além disso, familiares de crianças com DEA demonstraram maiores preocupações em relação à criança, o que afetou a qualidade de vida familiar, inclusive nos domínios relacionados também aos aspectos físicos ${ }^{10}$.

O esforço dispensado pela mãe de uma criança com DEA, para descobrir a mensagem comunicativa que seu filho lhe dirige, manifesta-se em insistentes tentativas de se aproximar da criança na busca por compreendê-la ${ }^{11}$. Tais crianças são menos propensas a apresentar sorrisos e contato visual com intenção comunicativa em relação às suas mães, do que as crianças normais ${ }^{7}$.

Nas famílias de crianças autistas pode-se encontrar comunicação pouco clara, menos investida de carga emocional adequada, pouco espaço para a expressão de agressividade e afeição física ${ }^{6}$ do que em famílias de crianças com síndrome de Down e normais. Nesse sentido, pesquisas tem investigado influencias das interações maternas no desenvolvimento de crianças com DEA ${ }^{6,12}$.

Essas interações certamente estão relacionadas aos prejuízos que tais crianças manifestam no uso pragmático da comunicação ${ }^{13-15}$; aspecto que interfere significantemente em suas relações sociais ${ }^{14,16}$; e em alterações de comportamento ${ }^{17 .}$ Habilidades pragmáticas incluem a intenção comunicativa ${ }^{18}$; a manutenção da atenção do ouvinte e a articulação clara da mensagem, o uso espontâneo da linguagem, a coerência discursiva, o uso de diferentes estilos linguísticos de acordo com o contexto e interlocutores, o reparo das quebras comunicativas e a inclusão de informações, quando o ouvinte não compreende a mensagem ${ }^{18}$.

Portanto, análises pragmáticas das interações entre crianças com DEA e suas mães, são fundamentais para a compreensão e intervenção desses casos $^{5,15,19}$. Pesquisadores apontam que o prognóstico de desenvolvimento da linguagem em crianças com DEA está mais significantemente associado às suas iniciativas de comunicação do que as respostas emitidas pelas mesmas ${ }^{20}$. Nesse sentido, estudos sobre tal temática podem contribuir para a reflexão e elaboração de medidas de intervenção preventiva e terapêutica em fonoaudiologia, direcionadas para pais e cuidadores, que favoreçam o prognóstico e qualidade de vida das crianças e suas mães.

Assim, o objetivo deste estudo foi investigar as iniciativas de comunicação na interação entre crianças com distúrbios do espectro autístico (DEA) e suas mães, por meio de uma análise pragmática.

\section{MÉTODO}

Foi realizado um estudo transversal com 20 participantes, sendo cinco crianças com diagnósticos incluídos nos DEA, estabelecidos segundo critérios específicos ${ }^{21,22}$, de ambos os gêneros, idades entre cinco e 12 anos e suas respectivas mães (Tabela 1). Para compor o grupo controle participaram cinco crianças com desenvolvimento normal e suas respectivas mães, pareadas de acordo com as idades e gênero das crianças com DEA.

As crianças com DEA recebiam atendimento fonoaudiológico em uma clínica-escola de uma cidade de médio porte do interior paulista. Os critérios de inclusão para a participação das mães nesta pesquisa foram: ser alfabetizada; ser mãe biológica da criança e ser responsável pelos cuidados principais com a criança participante do estudo. 
Tabela 1 - Caracterização das crianças com distúrbios do espectro autístico e de suas mães

\begin{tabular}{|c|c|c|c|c|c|c|c|c|}
\hline \multirow[b]{2}{*}{ Díades } & \multicolumn{4}{|c|}{ Dados das mães } & \multicolumn{4}{|c|}{ Dados das crianças } \\
\hline & $\begin{array}{l}\text { Idade } \\
\text { (anos) }\end{array}$ & Estado civil & Escolaridade & Profissão & $\begin{array}{l}\text { Idade } \\
\text { (anos) }\end{array}$ & Gênero & $\begin{array}{c}\text { Diagnóstico } \\
\text { médico }\end{array}$ & Escolaridade \\
\hline 1 & 34 & Divorciada & $\begin{array}{l}2^{\circ} \text { grau } \\
\text { completo }\end{array}$ & Vendedora & 5 & masculino & Autismo & Pré \\
\hline 2 & 52 & Casada & $\begin{array}{c}1^{\circ} \text { grau } \\
\text { incompleto }\end{array}$ & Cozinheira & 12 & masculino & Autismo & APAE \\
\hline 3 & 55 & Casada & $\begin{array}{l}1^{\circ} \text { grau } \\
\text { completo }\end{array}$ & $\begin{array}{l}\text { Enfermeira } \\
\text { aposentada }\end{array}$ & 7 & feminino & TID & $1^{\circ}$ ano \\
\hline 4 & 41 & Casada & $\begin{array}{c}1^{\circ} \text { grau } \\
\text { incompleto }\end{array}$ & Doméstica & 11 & masculino & TID & $4^{\circ}$ ano \\
\hline 5 & 35 & Divorciada & 3o grau & Professora & 11 & feminino & Autismo & 60 ano \\
\hline
\end{tabular}

Legenda: $\mathrm{TID}=$ Transtorno invasivo do desenvolvimento

Os materiais utilizados neste estudo foram: equipamento convencional de filmagem, objetos lúdicos como bola, fantoches, carrinhos, utensílios domésticos, boneca e revistas para a interação. Os instrumentos utilizados foram: uma Ficha informativa; a Escala de Avaliação de Traços Autísticos (ATA) e, para a análise das situações de comunicação entre as crianças e suas mães, empregou-se o Protocolo de Pragmática ${ }^{23}$, que mensura e categoriza as iniciativas de comunicação, os meios utilizados (verbal, vocal e gestual) e as funções comunicativas expressas.

\section{Procedimentos}

Para a caracterização dos participantes foi elaborada uma Ficha informativa especificamente para esta pesquisa que consta de questões abertas e fechadas, que constava informações sobre: identificação das crianças e das mães sobre idade, gênero, grau de escolaridade, entre outros aspectos.

Embora as crianças com DEA tivessem seus diagnósticos estabelecidos por profissionais especializados, a fim de caracterizar melhor a amostra, foi utilizada a Escala de Avaliação de Traços Autísticos - ATA ${ }^{24}$. Este instrumento é composto por 23 sub-escalas, cada uma dividida em diferentes itens. Sua construção foi realizada considerandose os critérios diagnósticos do DSM-III, DSM-III$\mathrm{R}$ e da CID-10 e, na padronização dos autores foram utilizadas também as correções de critérios decorrentes da publicação do DSM-IV. A ATA é um instrumento de fácil aplicação, por um profissional conhecedor do quadro, embora não necessariamente médico, sendo este o responsável pela avaliação das respostas dadas em função de cada item ${ }^{24}$. Não é portanto, uma entrevista diagnóstica, mas uma prova estandardizada que aponta o perfil de conduta da criança, auxiliando também na elaboração de um diagnóstico mais confiável.
A escala é pontuada baseando-se nos seguintes critérios: cada sub-escala da prova tem um valor de 0 a 2; a pontuação zero é indicada se não houver a presença de nenhum item, 1 se houver apenas um item e 2 se houver mais de um item, realizando-se uma soma aritmética dos pontos obtidos. Seu ponto de corte é de vinte e três ${ }^{24}$.

Para a investigação das interações entre as crianças e suas mães, foram realizadas filmagens de 30 minutos de interação lúdica das díades mãecriança em suas próprias residências. A análise das filmagens foi realizada por meio do Protocolo de Pragmática, que consiste na caracterização dos atos comunicativos apresentados pelo indivíduo em uma situação de interação. Visa registrar os dados que reflitam a efetividade e competências comunicativas da criança e caracterizá-los, baseando-se em três critérios específicos: 1) número de atos por minuto - reflete a iniciativa comunicativa, pois é calculado pela quantidade de atos iniciados pela criança durante a interação com o adulto; 2) meio comunicativo predominante - reflete a competência verbal, pois é analisado pela quantidade de uso dos meios verbal, vocal e/ou gestual, nos atos apresentados pela criança; 3) função comunicativa predominante - reflete a competência funcional, pois é caracterizada pelo tipo de função social que os atos realizados pela criança representam. A transcrição desses critérios é primeiramente registrada no Protocolo para a transcrição da fita e posteriormente na Ficha síntese do perfil comunicativo.

Neste estudo foi considerada a divisão das funções comunicativas entre funções mais e menos interativas ${ }^{25}$, sendo entendidos como mais interativos os atos dirigidos às mães, aguardando respostas; e menos interativos os atos não dirigidos a mães e que, deste modo não aguardam respostas. São, portanto, consideradas mais 
interativas as funções: pedido de objeto, pedido de ação, pedido de rotina social, pedido de consentimento, pedido de informação, reconhecimento do outro, exibição, comentário, nomeação, exclamativa, narrativa, expressão de protesto, protesto e jogo compartilhado. As funções menos interativas são: reativa, performativa, não-focalizada, jogo, exploratória e auto-regulatória.

Esta pesquisa obteve aprovação do Comitê de Ética e pesquisa da Faculdade de Filosofia e Ciências - UNESP - Marília, protocolo n. 3628/2008. Foi solicitado às mães participantes o preenchimento do termo de consentimento livre e esclarecido para a participação no estudo.

Foram realizadas análises estatísticas dos resultados, sendo os dados numérico-estatísticos apresentados por meio de médias, variações e desvios padrão. Para comparação dos achados entre as crianças com DEA e as crianças do grupo controle, foi utilizado o teste não-paramétrico de Wilcoxon; e para a comparação entre as mães de ambos os grupos empregou-se o teste não-paramétrico de Mann-Whitney ${ }^{26}$. Foi utilizada a medida descritiva $P$, adotando-se o nível crítico de significância de $5 \%$ $(p<0,05)$.

\section{RESULTADOS}

Primeiramente são apresentados os dados sobre as crianças com DEA, referentes ao perfil de manifestações clínicas, obtidos por meio da aplicação da Escala de Avaliação de Traços Autísticos ${ }^{24}$.

Tabela 2 - Síntese dos resultados obtidos a partir da escala de avaliação de traços autísticos

\begin{tabular}{cc}
\hline Díades & $\begin{array}{l}\text { Escores das crianças } \\
\text { do espectro autístico }\end{array}$ \\
\hline 1 & 37 \\
2 & 42 \\
3 & 39 \\
4 & 31 \\
5 & 39 \\
\hline
\end{tabular}

Como mostra a Tabela 2, verificou-se que na Escala de Avaliação de Traços Autísticos as crianças com DEA das díades 1, 2, 3, 4 e 5, apresentaram escore de 37,0 - 42,0 - 39,0 - 31 e 39, respectivamente. Assim, crianças deste estudo demonstraram escores acima da nota de corte $(23,0)$, isto é, uma pontuação que caracteriza a presença de transtorno invasivo do desenvolvimento em todos os casos.

No que se refere à análise pragmática das interações entre as crianças e suas mães, a Tabela 3 ilustra o número de atos comunicativos utilizados por minuto pelas crianças com DEA e seus pares normais, indicando suas médias, medianas e desvios-padrão.

Nas comparações entre as crianças com DEA e normais, verificou-se que as crianças com DEA (média $=4,05$ ) apresentaram significantemente menos iniciativas comunicativas por minuto do que as crianças normais (média=7,45) (Tabela 3).

Tabela 3 - Valores das médias, medianas e desvios-padrão do número de iniciativas de comunicação realizadas por minuto pelas crianças

\begin{tabular}{ccc}
\hline $\begin{array}{c}\text { Díades - } \\
\text { crianças }\end{array}$ & $\begin{array}{c}\text { Espectro } \\
\text { autístico }\end{array}$ & Grupo controle \\
\hline 1 & 2,1 & 7,5 \\
2 & 2,5 & 6,9 \\
3 & 4,7 & 5,3 \\
4 & 5,1 & 8,7 \\
5 & 5,8 & 8,8 \\
\hline Média & 4,0 & 7,4 \\
Mediana & 4,7 & 7,5 \\
Desvio-padrão & 1,6 & 1,4 \\
\hline
\end{tabular}

Quanto aos meios comunicativos, a Tabela 4 apresenta as médias, medianas e desvios-padrão dos meios verbal, vocal e gestual, utilizados pelas crianças com DEA e seus pares normais para iniciar a comunicação com suas mães. 
Tabela 4 - Valores das médias, medianas e desvios-padrão dos meios comunicativos utilizados pelas crianças

\begin{tabular}{ccccccc}
\hline \multirow{2}{*}{$\begin{array}{c}\text { Díades- } \\
\text { crianças }\end{array}$} & \multicolumn{2}{c}{ Verbal } & \multicolumn{2}{c}{ Vocal } & \multicolumn{2}{c}{ Gestual } \\
\cline { 2 - 7 } & $\begin{array}{c}\text { Espectro } \\
\text { autístico }\end{array}$ & $\begin{array}{c}\text { Grupo } \\
\text { controle }\end{array}$ & $\begin{array}{c}\text { Espectro } \\
\text { autístico }\end{array}$ & $\begin{array}{c}\text { Grupo } \\
\text { controle }\end{array}$ & $\begin{array}{c}\text { Espectro } \\
\text { autístico }\end{array}$ & $\begin{array}{c}\text { Grupo } \\
\text { controle }\end{array}$ \\
\hline 1 & 0 & 70,0 & 2,0 & 13,0 & 29,0 & 80,0 \\
2 & 0 & 95,0 & 10,0 & 7,0 & 42,0 & 75,0 \\
3 & 59,0 & 59,0 & 9,0 & 5,0 & 10,0 & 52,0 \\
4 & 71,0 & 100,0 & 5,0 & 10,0 & 28,0 & 32,0 \\
5 & 71,0 & 105,0 & 15,0 & 2,0 & 7,0 & 32,0 \\
\hline Média & 2,9 & 5,7 & 0,5 & 0,5 & 1,5 & 3,6 \\
Mediana & 3,9 & 6,3 & 0,6 & 0,5 & 1,9 & 3,5 \\
Desvio- & 37,0 & 20,1 & 4,9 & 4,3 & 14,5 & 22,8 \\
padrão & & & & & & \\
\hline$p<0,05-$ Teste de Wilcoxon & & & & &
\end{tabular}

Conforme a Tabela 4, as crianças com DEA demonstraram significantemente menos meios verbais (média $=2,9$ ) e gestuais (média $=1,5$ ) do que o grupo controle (média=5,7 e média $=3,6$ respectivamente). Em relação ao meio vocal, não há diferenças estatisticamente significantes entre as crianças com DEA (média $=0,5$ ) e as normais (média $=0,5)$.
Na Tabela 5, é possível observar as médias, medianas e desvios-padrão das funções comunicativas mais e menos interativas utilizadas pelas crianças com DEA e seus pares normais durante as situações de interação com suas mães.

Tabela 5 - Valores das médias, medianas e desvios-padrão das funções mais e menos interativas utilizadas pelas crianças durante as situações filmadas

\begin{tabular}{|c|c|c|c|c|}
\hline \multirow[b]{2}{*}{ Díades - Crianças } & \multicolumn{2}{|c|}{ Funções mais interativas } & \multicolumn{2}{|c|}{ Funções menos interativas } \\
\hline & $\begin{array}{l}\text { Espectro } \\
\text { autístico }\end{array}$ & Grupo controle & $\begin{array}{l}\text { Espectro } \\
\text { autístico }\end{array}$ & Grupo controle \\
\hline 1 & 12,0 & 104,0 & 19,0 & 9,0 \\
\hline 2 & 16,0 & 104,0 & 22,0 & 0 \\
\hline 3 & 25,0 & 74,0 & 46,0 & 6,0 \\
\hline 4 & 63,0 & 130,0 & 14,0 & 0 \\
\hline 5 & 76,0 & 127,0 & 11,0 & 5,0 \\
\hline Média & 2,6 & 7,2 & 1,5 & 0,3 \\
\hline Mediana & 1,7 & 6,9 & 1,3 & 0,3 \\
\hline Desvio-padrão & 29,1 & 22,5 & 13,9 & 3,9 \\
\hline
\end{tabular}

$p<0,05$ - Teste de Wilcoxon

As crianças com DEA e as crianças normais apresentaram diferenças estatisticamente significantes no uso das funções comunicativas. Em relação às funções comunicativas mais interativas, as crianças com DEA apresentaram menor frequência (média=2,6) do que as normais (média=7,2). Já as funções menos interativas expressas pelas crianças com DEA, apresentaram maior ocorrência (média $=1,5$ ) do que as demonstradas pelas crianças normais (média $=0,3$ ) (Tabela 5).

Em relação às mães, a Tabela 6 ilustra o número de iniciativas de comunicação realizadas por minuto pelas mães das crianças com DEA e as mães do grupo controle, indicando suas médias, medianas e desvios-padrão. 
Tabela 6 - Valores das médias, medianas e desvios-padrão do número de iniciativas de comunicação realizadas por minuto pelas mães

\begin{tabular}{ccc}
\hline Díades - mães & $\begin{array}{c}\text { Espectro } \\
\text { autístico }\end{array}$ & Grupo controle \\
\hline 1 & 4,2 & 9,1 \\
2 & 4,1 & 8,0 \\
3 & 4,0 & 5,8 \\
4 & 3,3 & 6,5 \\
5 & 5,9 & 7,4 \\
\hline Média & 4,3 & 7,4 \\
Mediana & 4,1 & 7,4 \\
Desvio-padrão & 0,9 & 1,3 \\
\hline
\end{tabular}

$p<0,05-$ Teste de Mann-Whitney

Nas comparações entre as mães de crianças com DEA e as mães do grupo controle, verificouse que no grupo com DEA (média=4,3) as mães apresentaram significantemente menos iniciativas comunicativas por minuto do que as mães das crianças normais (média $=7,4$ ) (Tabela 6 ).

\section{DISCUSSÃO}

Este estudo possibilitou mensurar e categorizar as iniciativas de comunicação na interação entre crianças com DEA e suas mães, por meio de uma análise pragmática. Assim, foram investigadas: a quantidade de iniciativas comunicativas apresentadas por minuto pelas crianças e suas mães, os meios comunicativos utilizados para expressá-las e a frequência de funções mais e menos interativas manifestadas pelas crianças.

Investigações desta natureza, com ênfase em aspectos pragmáticos, durante interações entre crianças com DEA e suas mães, são fundamentais para a compreensão e intervenção desses $\operatorname{casos}^{19}$. Como explicaram diversos autores, os prejuízos em habilidades pragmáticas são as principais dificuldades em comunicação para crianças com DEA ${ }^{13-15}$ e influenciam diretamente suas relações sociais ${ }^{14,16}$ e alterações comportamentais ${ }^{17}$.

Nesta pesquisa foram também analisados os perfis comportamentais das crianças com DEA, a partir do emprego da Escala de Avaliação de Traços Autísticos ${ }^{24}$. Conforme a literatura, as diferenças individuais entre as crianças estão presentes e afetam as características maternas ${ }^{4}$. As iniciativas de comunicação das mães de crianças com DEA podem estar associadas ao nível de desenvolvimento da linguagem ${ }^{4,27}$ e com a intensidade de problemas comportamentais apresentados pela criança ${ }^{27}$.
Os resultados demonstraram que todas as crianças com DEA apresentaram perfis de comportamento que dificultaram as relações interpessoais. Os dados aqui encontrados sobre as características de crianças com DEA concordam com as descrições da literatura, que referem atraso, ou ausência total de desenvolvimento da linguagem falada ${ }^{21}$; comprometimento acentuado da capacidade de iniciar ou manter conversação, $0^{5,21}$; uso estereotipado e repetitivo da linguagem ${ }^{21}$; notável pobreza na comunicação não-verbal envolvendo gestos, prosódia inadequada ao conteúdo do diálogo ${ }^{5,18}$; e repertório lexical alterado ${ }^{28 ;}$ aspectos que são também envolvidos nas habilidades pragmáticas de um indivíduo ${ }^{18}$.

Nas interações com as mães, as crianças com DEA deste estudo apresentaram menos iniciativas de comunicação do que as crianças normais, conforme os achados de outros autores ${ }^{12}$. Tais dados mostram que, mesmo que de maneira não convencional, as crianças com DEA foram capazes de se comunicar, o que está de acordo com as afirmações da literatura ${ }^{13-15}$. Verificou-se, ainda, variabilidade quanto ao número das iniciativas de comunicação realizadas pelas crianças com DEA, o que indica certa heterogeneidade em seus perfis comunicativos, como observado por outros autores ${ }^{15}$.

Quanto aos meios comunicativos, quando comparados, as crianças com DEA e as normais, apresentaram diferenças significantes no uso dos meios comunicativos, sendo que as crianças com DEA mostraram estatisticamente menor uso dos meios verbais e gestuais do que as crianças normais.

Ao examinar cada grupo de crianças separadamente, verificou-se que o grupo controle utilizou principalmente o meio verbal, caracterizado pelo uso de palavras inteligíveis. Já o grupo de crianças com DEA demonstraram heterogeneidade também na utilização dos meios comunicativos. Sabe-se que o meio comunicativo preponderante na faixa etária das crianças desta pesquisa é o meio verbal, geralmente acompanhado pelo gestual ${ }^{29}$. Em torno de 18 meses, as crianças com desenvolvimento de linguagem normal vão substituindo gestos, vocalizações, idiossincrasias, por palavras propriamente ditas ${ }^{30}$. Portanto os achados desta pesquisa estão de acordo com a literatura, no que se refere tanto aos meios utilizados pelo grupo de crianças normais ${ }^{29}$ como pelo grupo de crianças com DEA ${ }^{15}$.

A investigação dos meios comunicativos e mais especificamente do meio gestual em crianças com DEA é importante, pois aspectos da comunicação como o contato visual e a atenção conjunta, estão envolvidos em seu desenvolvimento posterior de linguagem ${ }^{16}$. Como enfatizou a literatura ${ }^{30}$,quando 
a criança não faz uso de estruturas linguísticas, ela pode se comunicar de diversas formas, como: gestos indicativos e representativos, vocalizações articuladas e não articuladas, idiossincrasias; formas estas associadas entre si, ou a algumas poucas palavras isoladas ${ }^{30}$. Assim, mesmo que as crianças com DEA deste trabalho não tenham utilizado preferencialmente o meio verbal para se expressar, seria possível o estabelecimento da comunicação com suas mães, dependendo das funções comunicativas utilizadas. Como exemplificado na literatura $^{30}$, quando uma criança dirige-se à porta e pára diante dela, não há indícios de intencionalidade no seu comportamento (por mais que se tenha certeza de que ela quer sair). Mas, se ela dirige-se à porta, pára diante dela, olha para alguém, aponta para a porta e olha para alguém novamente, então, há indícios de comportamento intencional. O "dirigir-se ao outro" é fundamental para garantir a presença de intencionalidade na comunicação.

No que se refere às funções comunicativas, foram observadas diferenças estatisticamente significantes ao comparar as crianças com DEA $e$ as normais. As crianças com DEA apresentaram maior uso de comunicação menos interativa do que as crianças normais, concordando com outros autores ${ }^{12}$. Estes resultados confirmam as dificuldades das crianças com DEA quanto ao uso funcional da comunicação ${ }^{13,15}$; e indicam que, embora tenham expressado comunicação, em sua maior parte ela não foi especificamente dirigida às suas mães. No grupo de crianças normais, foi encontrado maior uso das funções mais interativas do que no grupo das crianças com DEA, independente da idade e do diagnóstico, o que corrobora com os achados de vários autores ${ }^{7,12}$.

Quanto às mães, foi observado que, como os filhos, as mães das crianças com DEA deste estudo também iniciaram menos a comunicação do que as mães do grupo controle. Estes resultados estão de acordo com os achados de outras pesquisas ${ }^{6,7,11,12}$, os quais também apontaram as dificuldades na comunicação entre crianças com DEA e suas mães.

Tais dados podem relacionar-se ao fato de as crianças com DEA terem manifestado predomínio de meios gestuais e funções menos interativas, o que pode dificultar as situações de interação e restringir a motivação de suas mães para iniciarem a comunicação. Estas colocações estão de acordo com os resultados de outras pesquisas que, observaram que crianças com DEA apresentam menos sorrisos e contato visual com intenção comunicativa quando em interação com suas mães, do que as crianças normais ${ }^{7}$.

Como destacou a literatura, a reciprocidade é imprescindível em qualquer situação de comunicação ${ }^{30}$, sendo que há relações de interdependência entre os comportamentos da mãe e da criança ${ }^{4}$.

A mãe de uma criança com DEA precisa despender grande esforço na busca por entender as mensagens do filho, com insistentes tentativas de se aproximar e compreendê-la ${ }^{11}$. Destaca-se que as emissões gestuais e vocais são caracterizadas por segmentos ininteligíveis ao interlocutor, comprometem a situação dialógica natural presente no contexto comunicativo, dificultam a compreensão da mensagem e a interpretação comunicativa do falante ${ }^{29}$. Na presente pesquisa, esses fatos podem ter implicado dificuldades na compreensão e interpretação das emissões das crianças com DEA por parte de suas mães, o que pode estar relacionado com o perfil comunicativo apresentado pelas mesmas.

Entretanto, mesmo que a criança tenha sua parcela de responsabilidade nas interações pouco eficazes, cabe ao adulto, que em princípio, tem mais discernimento e maturidade para buscar relações mais efetivas e o papel de modificar as interações ${ }^{30}$. Em qualquer díade mãe-criança, o fluxo da interação requer um permanente ajustamento, até se estabelecer uma sincronia entre ambos ${ }^{11}$.

Assim, sugere-se que é importante o empenho das mães em descrever e interpretar a comunicação de seus filhos com DEA para que consigam compreender suas tentativas de entendimento mútuo e propiciem circunstâncias favoráveis para a criança fazer o melhor uso possível de seus meios comunicativos. Estes aspectos, evidentemente devem ser também investigados e levados em consideração pelos profissionais das áreas da saúde e da educação, na elaboração de procedimentos de intervenção com crianças portadoras de DEA. Compete, portanto, aos profissionais oferecer respaldo às famílias, a fim de obter um melhor desempenho com seus filhos com DEA. Como destacou a literatura, é necessário o apoio de outros familiares e o respaldo profissional oferecido à criança e sua família ${ }^{27}$ no sentido de promover melhor qualidade de vida familiar.

\section{CONCLUSÃO}

O presente estudo permitiu construir um paralelo entre a iniciativa e a intenção comunicativa das mães e crianças com DEA e aquelas com desenvolvimento normal e verificar aspectos da discrepância na quantidade e qualidade dessas interações. Os resultados obtidos demonstram diferenças significantes entre as iniciativas comunicativas das crianças com DEA e suas mães, quando comparadas ao grupo controle. As díades que envolveram 
crianças com DEA iniciaram menos a comunicação e utilizaram predominantemente gestos e vocalizações menos interativas.

Estes dados permitiram descrever aspectos fundamentais para a compreensão das manifestações clínicas da população estudada e forneceram elementos para auxiliar na investigação diagnóstica, na elaboração de condutas de prevenção e intervenção terapêutica fonoaudiológica. Mais especificamente, as implicações terapêuticas destes achados consistem em possibilitar a estruturação de contextos terapêuticos e planejamento também de atividades direcionadas às necessidades específicas de cada família e também orientar estes familiares a respeito de seu papel na busca por interações mais efetivas, prazerosas e com atribuição de sentido às iniciativas das crianças. Este tipo de investigação permite ainda que os profissionais orientem-se pelas iniciativas bem sucedidas realizadas pelas mães de seus pacientes.

\begin{abstract}
Purpose: to investigate the communication initiatives among children with autistic spectrum disorders and their mothers, employing a pragmatic analysis. Method: five children with autistic spectrum disorders, both genders, ages ranging between five and 12-year and their mothers. The control group comprised 5 children with normal development, paired with children with autistic spectrum disorders, according to gender and age and their respective mothers, totaling 20 participants. For the characterization of the sample, an Information Form and a Scale for the Assessment of Autistic Behavior were employed. Each dyad was filmed for 30 minutes during ludic interactions and analyzed according to the Pragmatic Protocol. This instrument evaluates communication initiatives, means and communicative functions. Data were analyzed statistically $(p<0.05$; non-parametric tests of Wilcoxon and Mann-Whitney were employed). Results: statistically significant differences were observed between the communicative initiatives of children with autistic spectrum disorders and their mothers, when compared to the control group. The dyads which involved children with autistic spectrum disorders showed few communication initiatives and used predominantly gestures and less interactive vocalization. Conclusion: such data turned easier the description of the fundamental aspects for the comprehension of the clinical manifestations in the studied population and also for elaborating intervention programs aiming at improving the quality of life related to the children and their respective families.
\end{abstract}

KEYWORDS: Mother-Child Relations; Autistic Disorder; Communication; Intervention Studies

\section{REFERÊNCIAS}

1. Pajulo M, Pyykkönen N, Kalland M, Sinkkonen $J$, Helenius H, Punamäki RL. Substance abusing mothers in residential treatment with their babies: postnatal psychiatric symptomatology and its association with mother-child relationship and later need for child protection actions. Nord J Psychiatry. 2010; Jun 30, Epub ahead of print.

2. Kawai M, Namba K, Yato Y, Negayama K, Sogon $\mathrm{S}$, Yamamoto H. Developmental trends in motherinfant interaction from 4-months to 42-months: using an observation technique. Japan Children's Study Group. J Epidemiol. 2010; 20(2):427-34.

3. Yato $Y$, Tanaka D, Shinohara R, Sugisawa $Y$, Tanaka E, Tong L, Yamakawa N, Anme T, Kawai M, Maeda T. Infant responses to maternal still face at 9 months predict social abilities at 18 months.
Japan Children's Study Group. J Epidemiol. 2010; 20(2):435-40.

4. Zamberlan MAT. Interação mãe-criança: enfoques teóricos e implicações decorrentes de estudos empíricos. Estudos de Psicologia. 2002; 7(2):399-406.

5. Klin A, Saulnier C, Sparrow S, Cicchetti D, Volkmar F, Lord C. Social and communication abilities and disabilities in higher functioning individuals with autism spectrum disorders: the vineland and the ADOS. Journal of Autism and Developmental Disorders. 2007; 37:748-59.

6. Sprovieri MHS, Assumpção Jr FB. Dinâmica familiar de crianças autistas. Arq. Neuro-Psiquiatr. 2001;59(2A):230-7.

7. Dawson G, Hill D, Spencer A, Galpert L, Watson L. Affective exchanges between young autistic children and theirs mothers. J. Abnorm. Chil Psychol. 1990; 3:335-45 
8. Mazefsky CA, Conner CM, Oswald DP. Association between depression and anxiety in high-functioning children with autism spectrum disorders and maternal mood symptoms. Autism Res. 2010;3(3):120-7.

9. Ekas N, Whitman TL. Autism symptom topography and maternal socioemotional functioning. Am J Intellect Dev Disabil. 2010;115(3):234-49.

10. Shu BC. Quality of life of family caregivers of children with autism: the mother's perspective. Autism. 2009;13(1):81-91.

11. Roncon P. Abordagens familiares face ao autismo. Análise Psicológica. 2003; 1(XXI):53-7.

12. Warreyn P., Roeyers H, De Groote I. Early social communicative behaviours of preschoolers with autism spectrum disorder during interaction with their mothers. Autism. 2005;9(4):342-61.

13. Misquiatti, ARN, Brito, MC. Terapia de linguagem de irmãos com transtornos invasivos do desenvolvimento: estudo longitudinal. Rev Soc Bras Fonoaudiolo. 2010; 15(1):134-9.

14. Volden J, Coolican J, Garon N, White J, Bryson S. Brief report: pragmatic language in autism spectrum disorder: relationships to measures of ability and disability. J Autism Dev Disord. 2009;39(2):388-93.

15. Fernandes FD, Cardoso C, Sassi FC, Amato $\mathrm{CH}$, Sousa-Morato PF. Fonoaudiologia e autismo: resultado de três diferentes modelos de terapia de linguagem. Pró-Fono Revista de Atualização Científica. 2008;20(4):267-72

16. Drew A, Baird G, Taylor E, Milne E, Charman T. The social communication assessment for toddlers with autism (SCATA): an instrument to measure the frequency, form and function of communication in toddlers with autism spectrum disorder. J Autism Dev Disord. 2007; 37:648-66.

17. Ketelaars MP, Cuperus J, Jansonius K, Verhoeven L. Pragmatic language impairment and associated behavioural problems. Int $\mathrm{J}$ Lang Commun Disord. 2009; 28:1.

http://dx.doi.org/10.1590/S1516-18462011005000052

RECEBIDO EM: 22/09/2010

ACEITO EM: 23/12/2010

Endereço para correspondência:

Andréa Regina Nunes Misquiatti

Av. Hygino Muzzi Filho, n. 737

Caixa Postal: 181

Marília - SP

CEP:17525-050

E-mails: brito_mariaclaudia@yahoo.com

amisquiatti@uol.com.br
18. Landa R. Early communication development and intervention for children with autism. Mental Retardation and Developmental Disabilities Research Reviews. 2007; 3:16-25.

19. Rutherford MD; Young GS; Hepburn S; Rogers SJ. A longitudinal study of pretend play in autism. J Autism Dev Disord. 2007; 37(6):1024-39.

20. Charman T, Baron-Cohen S, Swettenham J, Baird G, Drew A, Cox A. Predicting language outcome in infants with autism and pervasive developmental disorder. International Journal of Language and Communication Disorders. 2003; 38:265-85.

21. American Psychiatry Association. Manual diagnóstico estatístico de transtornos mentais. (DSM-IV-TR). Porto Alegre: Artes Médicas; 2002.

22. Organização Mundial de Saúde. Classificação de transtornos mentais de comportamento- CID-10Critérios diagnósticos para pesquisa. Porto Alegre: Artes Médicas; 1993; 10 ed.

23. Fernandes FDM. Pragmática. In: Andrade CRF, Befi-Lopes DM, Fernandes FDM, Wertzner HF. ABFW: teste de linguagem infantil nas áreas de fonologia, vocabulário, fluência e pragmática. Carapicuiba: Pró-Fono; 2004.

24. Assumpção Jr FB, Baptista F, Gonçalves JDM, Cuccolichio S, Amorim LCD, Rego F, Gomes C, Falcão MS. Escala de avaliação de traços autísticos (ATA): segundo estudo de validade/Scale for the assessment of Autistic Behaviour (ATA) Med. reabil. 2008;27(2):41-4.

25. Wetherby AM, Prutting CA. Profiles of communicative and cognitive - social abilities in autistic children. Journal of Speech and Hearing Research. 1984; 27:364-77.

26. Siegel, S. Estatística não-paramétrica para as ciências do comportamento. McGraw-Hill; 1981.

27. Davis K, Gavidia-Payne S. The impact of child, family, and professional support characteristics on the quality of life in families of young children with disabilities. Journal of Intellectual \& Developmental Disability. 2009, 34:153-62.

28. Paul R, Orlovski SM, Marcinko HC, Volkmar F. Conversational behaviors in youth with highfunctioning ASD and Asperger syndrome. J Autism Dev Disord, 2009; 39:115-25.

29. Rodrigues A, Befi-Lopes D. Comparação entre as habilidades pragmáticas de crianças normais e crianças com alteração de desenvolvimento de linguagem. Rev Soc Bras Fonoaudiolo. 2004; 9(2):81-7.

30. Hage SRV. A avaliação fonoaudiológica em crianças sem oralidade. Tópicos em Fonoaudiologia. 2003; 5:175-85. 